\title{
lonization energy and electron affinity of a metal cluster in the stabilized jellium model: Size effect and charging limit
}

\author{
Michael Seidl and John P. Perdew \\ Department of Physics and Quantum Theory Group, Tulane University, New Orleans, Louisiana 70118 \\ Marta Brajczewska and Carlos Fiolhais \\ Departamento de Física da Universidade de Coimbra, P-3000 Coimbra, Portugal
}

(Received 31 October 1997; accepted 11 February 1998)

\begin{abstract}
We report the first reliable theoretical calculation of the quantum size correction $c$ which yields the asymptotic ionization energy $I(R)=W+\left(\frac{1}{2}+c\right) / R+O\left(R^{-2}\right)$ of a simple-metal cluster of radius $R$. Restricted-variational electronic density profiles are used to evaluate two sets of expressions for the bulk work function $W$ and quantum size correction $c$ : the Koopmans expressions, and the more accurate and profile-insensitive $\Delta \mathrm{SCF}$ expressions. We find $c \approx-0.08$ for stabilized (as for ordinary) jellium, and thus for real simple metals. We present parameters from which the density profiles may be reconstructed for a wide range of cluster sizes, including the planar surface. We also discuss how many excess electrons can be bound by a neutral cluster of given size. Within a continuum picture, the criterion for total-energy stability of a negatively charged cluster is less stringent than that for existence of a self-consistent solution. (C) 1998 American Institute of Physics. [S0021-9606(98)01819-4]
\end{abstract}

\section{INTRODUCTION}

The ionization energy $I$ of a spherical metal cluster approaches the work function $W$ of the planar metal surface as the cluster size tends to infinity. This is expressed by the asymptotic formula

$$
I(R)=W+\frac{\alpha}{R}+O\left(R^{-2}\right),
$$

where the cluster radius $R$ is usually defined by

$$
R=r_{s} N^{1 / 3}
$$

in terms of the number $N$ of valence electrons in the neutral cluster and the density parameter or Seitz radius $r_{s}$ of the corresponding bulk metal with average electron density

$$
\bar{n}=3 /\left(4 \pi r_{s}^{3}\right) \text {. }
$$

Within any classical theory, such as the spherical capacitor $^{1}$ or image-potential models, ${ }^{2,3}$ the size-effect coefficient $\alpha$ in Eq. (1) takes exactly the value $\frac{1}{2}$ (in atomic units where $e^{2}=\hbar=m=1, \quad \hbar^{2} / m e^{2}=1 \mathrm{bohr}, \quad$ and $m e^{4} / \hbar^{2}$ $=1$ hartree $=27.21 \mathrm{eV}$ ). A mathematical error in earlier presentations of the image-potential method, ${ }^{2}$ suggesting the wrong value $\frac{3}{8}$, has been uncovered and corrected recently. ${ }^{3}$ A more realistic approach needs to include quantum effects. These give rise to a quantum correction $c$ to the classical value, ${ }^{4-7}$

$$
\alpha=\frac{1}{2}+c .
$$

For simple metals treated in the jellium model, $c \approx-0.08,{ }^{5-8}$ for $2 \leqslant r_{s} \leqslant 6$ bohr, in good agreement with experimental data (as we shall see in Fig. 4; see also Ref. 6). In this case, $\alpha \approx 0.42$ by coincidence comes close to the arbitrary value $\frac{3}{8}$ resulting from the erroneous treatment of the image potentials. $^{2}$ Therefore this arbitrary value is sometimes still employed for the theoretical interpretation of experimental data. ${ }^{9}{ }^{10}$ Experiments on silver clusters, ${ }^{10}$ however, yield almost the value $\frac{1}{2}$ for $\alpha$, indicating that the quantum correction $c$ is close to zero for this nonsimple metal. For completeness, we note that the electron affinity of the neutral cluster (the binding energy of one excess electron) is ${ }^{4}$

$$
A(R)=W+\frac{-\frac{1}{2}+c}{R}+O\left(R^{-2}\right) .
$$

In previous work, ${ }^{8}$ we evaluated $c$ for the jellium model, but this model is not realistic for many of the simple metals, predicting an incorrectly negative surface energy ${ }^{11}$ for the metals of higher valence-electron density. In this article, we apply density functional theory ${ }^{12}$ to calculate $W$ and $c$ within a model for the simple metals that is much more realistic than jellium. We find $c \approx-0.08$ for this stabilized jellium model, ${ }^{13}$ as for ordinary jellium. The only inputs to this model are the bulk density $\bar{n}$ and the fact that the energy per electron in the bulk metal must minimize at this density, while the outputs are realistic surface properties for any simple metal. ${ }^{13-15}$ Like $c$ to which it is related, ${ }^{5}$ the curvature energy $a_{c}$ or size effect correction to the surface energy $a_{s}$ was previously found to be almost the same ${ }^{14}$ in stabilized as in ordinary jellium.

The stabilized jellium or structureless pseudopotential model ${ }^{13}$ starts from an expression for the total energy of a collection of valence electrons and close-packed ions, with the electron-ion interaction described by a local pseudopotential. The small terms of second- and higher-order in the pseudopotential are then dropped. The first-order term, effectively a constant potential over the interior of the metal, is adjusted to make the energy minimize at the given bulk den- 
sity $\bar{n}$. The surface properties of stabilized jellium are independent ${ }^{13}$ of the valence $z$, which we take to be 1 for simplicity.

In the following section, we recall several explicit expressions ${ }^{8}$ for $W$ and $c$ in the stabilized jellium model. The exact Koopmans and $\triangle \mathrm{SCF}$ expressions are equivalent for density profiles that solve the Euler equation $\delta E / \delta n(\mathbf{r})$ $=\mu$, but give results with significantly different accuracies for restricted-variational profiles. We also present parameters from which the density profiles may be reconstructed for a wide range of cluster sizes, including the planar surface, and show plots of the corresponding electron density profiles which are the only input to these expressions for $W$ and $c$. These profiles are obtained from restricted densityvariational calculations, described in Sec. III, where we also evaluate the expressions to obtain theoretical values for the work function $W$, the coefficient $c$, and the surface energy $a_{s}$ in the stabilized jellium model. By comparing to "direct" results from a fit to numerical ionization energies, we confirm that the "displaced-profile-change-in-self-consistentfield" $(\triangle \mathrm{SCF})$ expressions are by far the more profileinsensitive and accurate ones. The only previous calculations of $c$ for stabilized jellium ${ }^{16,17}$ were based on the lowaccuracy Koopmans expression; those early estimates are substantially revised here.

In Sec. IV, we apply our results for $W$ and $c$ to predict how many excess electrons can be bound by a cluster of a given size. Within a continuum picture such as the liquid drop model or the local density approximation, we find that the total-energy criterion for stability of a negatively charged cluster is less stringent than the condition for existence of a self-consistent solution. This observation is relevant to a recent controversy ${ }^{18,19}$ over the existence of negative ions within the local density approximation.

Our numerical results are compared to both results of self-consistent Kohn-Sham calculations and experimental ionization energies for small clusters in Sec. V. Our conclusions are summarized in Sec. VI.

\section{THEORETICAL EXPRESSIONS FOR $W$ AND $c$}

In both jellium ${ }^{11,20}$ and stabilized jellium, ${ }^{13}$ which are intended to model the simple metals, the work function $W$ and the quantum correction $c$ to the size-effect coefficient $\alpha$ can be expressed explicitly ${ }^{8}$ in terms of the electronic density profile $n(x)$ of the planar metal surface and its size-effect correction $f(x)$ in the expansion ${ }^{5}$

$$
n_{R}(x)=n(x)+\frac{f(x)}{R}+O\left(R^{-2}\right)
$$

of the density profile $n_{R}(x)$ of a neutral cluster with finite radius $R$. [The coordinate $x=r-R$ gives the distance from the edge of the spherical-jellium positive background. Integrals of $f(x)$ and $n(x)$ are related via Eq. (A5) of Ref. 5.] Figure 1(a) shows plots of these functions for stabilized jellium, obtained from the restricted variational calculations described in the next section. $n(x)$ approaches the bulk density $\bar{n}$ as $x \rightarrow-\infty$, and tends to zero as $x \rightarrow+\infty$. The function $f(x)$ must tend to zero in both directions $x \rightarrow \pm \infty$ far away
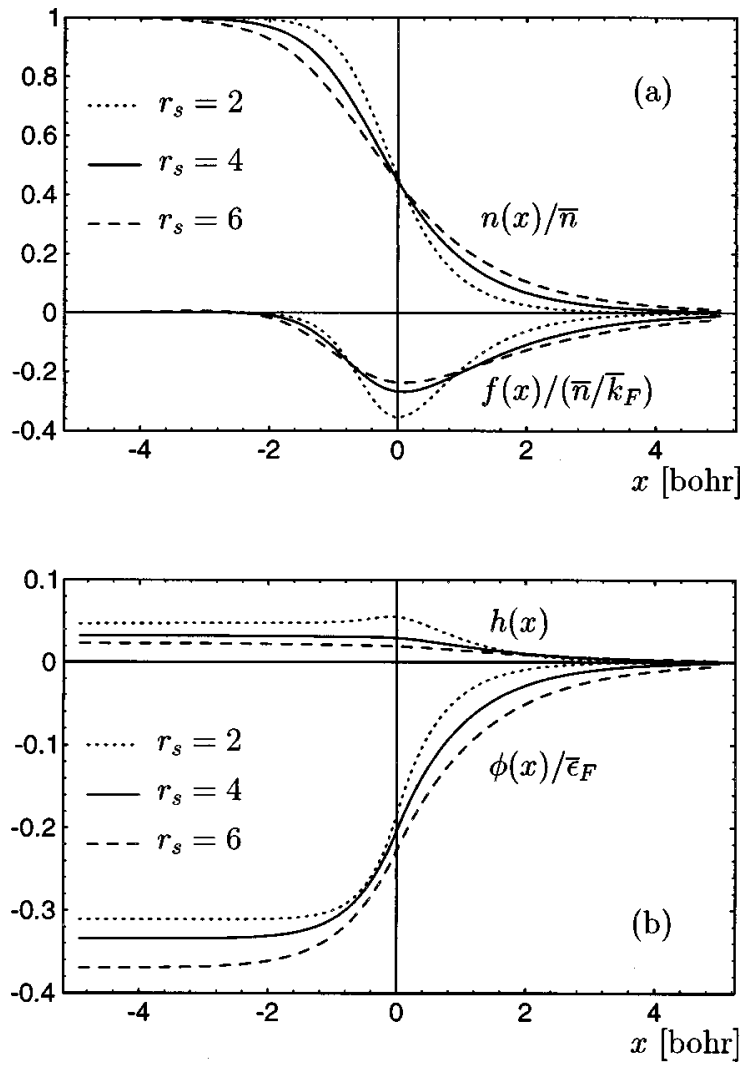

FIG. 1. (a) The functions $n(x)$ and $f(x)$ of Eq. (6) for stabilized jellium clusters at three different bulk densities, obtained from the variational calculations in Sec. III. $f(x)$ is given in units of $\bar{n} / \bar{k}_{F}$ where $\bar{k}_{F}$ $=(9 \pi / 4)^{1 / 3} / r_{s}$ is the bulk Fermi wave vector. (b) The functions $\phi(x)$ and $h(x)$ of Eq. (10), calculated for stabilized jellium clusters from the profiles in Fig. 1(a). $\phi(x)$, the electrostatic potential energy of an electron at the planar surface, is given in units of the bulk Fermi energy $\bar{\epsilon}_{F}=\frac{1}{2} \bar{k}_{F}^{2}$. The function $h(x)$ is dimensionless.

from the jellium edge $x=0$, because the density $n_{R}(x)$ of a large finite cluster approaches the bulk value $\bar{n}$ deep inside the cluster. We neglect self-compression effects. ${ }^{21}$

In the next section, we use these functions to calculate $W$ and $c$ from the " $\triangle \mathrm{SCF}$ " expressions ${ }^{22,23,7,8}$

$$
\begin{aligned}
W= & 4 \pi \int_{0}^{\infty} d x[x n(x)]-e_{k x c}(\bar{n})+\langle\delta v\rangle_{W S}\left[\frac{n(0)-\bar{n}}{\bar{n}}\right], \\
c= & 4 \pi \int_{0}^{\infty} d x\left[x^{2} n(x)+x f(x)\right]-\frac{2}{3} a_{s}[n] r_{s} \\
& +\langle\delta v\rangle_{W S}\left[\frac{f(0)}{\bar{n}}\right],
\end{aligned}
$$

where $e_{k x c}(\bar{n})$ is the noninteracting kinetic plus exchangecorrelation energy per electron in the homogeneous bulk metal of density $\bar{n}$. Eq. (7) from Ref. 13 and Eq. (8) from Ref. 8 are for stabilized jellium. ${ }^{13}$ For ordinary jellium, the terms containing the pseudopotential correction $\langle\delta v\rangle_{W S}=$ $-\bar{n} d e_{k x c}(\bar{n}) / d \bar{n}$ must be omitted, and the input profiles $n(x)$ and $f(x)$ must be recalculated with $\langle\delta v\rangle_{W S}=0$. In Eq. $(8)$, the surface energy is 


$$
a_{s}^{S J}[n]=a_{s}^{J}[n]+4 \pi r_{s}^{2}\langle\delta v\rangle_{W S} \int_{-\infty}^{0} d x[n(x)-\bar{n}] .
$$

The ordinary-jellium expression $a_{s}^{J}[n]$, e.g., Eq. (73) in Ref. 7 , is recovered by setting $\langle\delta v\rangle_{W S}=0$.

We can use the profiles $n(x)$ and $f(x)$ to calculate ${ }^{5,7}$ the electrostatic potential energy $\phi(x)$ of an electron at the planar metal surface and its size correction $h(x)$ in the expansion $^{5}$

$$
\phi_{R}(x)=\phi(x)+\frac{h(x)}{R}+O\left(R^{-2}\right)
$$

of the electrostatic potential of finite clusters with radius $R$. The results for stabilized jellium, obtained from the functions shown in Fig. 1(a), are plotted in Fig. 1(b). By convention, $\phi(+\infty)=h(+\infty)=0$. Furthermore, both the functions $\phi(x)$ and $h(x)$ approach constant values for $x \rightarrow-\infty$.

The electrostatic dipole barrier

$$
\Delta \phi=-\phi(-\infty)=4 \pi \int_{-\infty}^{\infty} d x\{x[n(x)-\bar{n} \Theta(-x)]\}
$$

of the planar surface and its size-effect correction

$$
\begin{aligned}
\Delta h=-h(-\infty)= & 4 \pi \int_{-\infty}^{\infty} d x\left\{x^{2}[n(x)-\bar{n} \Theta(-x)]\right. \\
& +x f(x)\}
\end{aligned}
$$

enter the "Koopmans" expressions ${ }^{8,11,16,17,23}$ for $W$ and $c$,

$$
\begin{aligned}
& W=\Delta \phi-\frac{d}{d \bar{n}}\left\{\bar{n} e_{k x c}(\bar{n})\right\}-\langle\delta v\rangle_{W S}, \\
& c=\Delta h .
\end{aligned}
$$

Again, as in the " $\triangle \mathrm{SCF}$ " expressions (7),(8) for stabilized jellium, the ordinary-jellium expressions are recovered by setting $\langle\delta v\rangle_{W S}=0$. Eq. (14) was derived in Ref. 16.

\section{DENSITY-VARIATIONAL CALCULATIONS IN THE STABILIZED JELLIUM MODEL}

As we have pointed out in Ref. 8, the $\triangle \mathrm{SCF}$ expressions (7),(8) are equivalent to the Koopmans expressions (13),(14) if evaluated with the correct functions $n(x)$ and $f(x)$, extracted from self-consistent ground-state densities $n_{R}(x)$ which solve the Euler equation for finite clusters. In contrast, if restricted-variational approximations to $n(x)$ and $f(x)$ are used, the Koopmans expressions are expected to yield less accurate results than the $\triangle \mathrm{SCF}$ expressions. The reason for this different behavior has been discussed in detail in Ref. 8, where we have also presented numerical data for the case of ordinary jellium. In the present work, we demonstrate this for the stabilized jellium model.

We employ the restricted density variational method of Ref. 24 with the parametrization

$$
n_{d, a, \gamma}(x)=\bar{n}\left[1+e^{(x-d) / a}\right]^{-\gamma}
$$

for the density profile. By varying the parameters $d, a$, and $\gamma$, we minimize the value $E_{R}\left[n_{d, a, \gamma}\right]$ of the density functional $^{12}$
TABLE I. Coefficients in expansions (19), etc., of the minimizing density parameters $d, a$, and $\gamma$ of the profile (15) in the stabilized jellium model. $r_{s}, d$, and $a$ are in bohr units.

\begin{tabular}{ccccccc}
\hline \hline$r_{s}$ & $d_{\infty}$ & $d_{1}$ & $a_{\infty}$ & $a_{1}$ & $\gamma_{\infty}$ & $\gamma_{1}$ \\
\hline 2 & -0.4238 & -0.185 & 0.3594 & -0.026 & 0.5453 & 0.108 \\
3 & -0.5918 & -0.194 & 0.4236 & -0.057 & 0.4978 & 0.056 \\
4 & -0.7249 & -0.187 & 0.4861 & -0.065 & 0.4795 & 0.051 \\
5 & -0.8421 & -0.216 & 0.5430 & -0.087 & 0.4685 & 0.027 \\
6 & -0.9801 & -0.245 & 0.6057 & -0.106 & 0.4565 & 0.010 \\
\hline \hline
\end{tabular}

$$
\begin{aligned}
E_{R}[n]= & T_{s}[n]+E_{x c}[n]+\frac{1}{2} \int d^{3} r \int d^{3} r^{\prime} \\
& \times \frac{\left[n(\mathbf{r})-n_{+R}(\mathbf{r})\right]\left[n\left(\mathbf{r}^{\prime}\right)-n_{+R}\left(\mathbf{r}^{\prime}\right)\right]}{\left|\mathbf{r}-\mathbf{r}^{\prime}\right|}+\Delta E_{R}^{S J}[n]
\end{aligned}
$$

for the ground-state energy of a cluster with radius $R$ $=r_{s} N^{1 / 3}$, subject to the normalization condition

$$
\int_{0}^{\infty} d r 4 \pi r^{2} n_{d, a, \gamma}(r-R)=N+\nu .
$$

(Here, for the case of charged clusters, $\nu$ is the number of excess electrons.) As in Ref. 7, we use for the noninteracting kinetic energy functional $T_{s}[n]$ the fourth-order gradient expansion of the extended Thomas-Fermi model. ${ }^{7}$ For an accurate description of the uniform density limit, we use the Perdew-Wang local-density approximation ${ }^{25}$ to the exchange-correlation energy functional $E_{x c}[n]$. In particular, in contrast to earlier work, ${ }^{7}$ we include the term

$$
\begin{aligned}
\Delta E_{R}^{S J}[n]= & \left(\epsilon_{M}+\bar{w}_{R}\right) \int d^{3} r n_{+R}(\mathbf{r}) \\
& +\langle\delta v\rangle_{W S} \int d^{3} r \Theta(R-r)\left[n(\mathbf{r})-n_{+R}(\mathbf{r})\right]
\end{aligned}
$$

in the functional (16), which turns ordinary jellium into stabilized jellium. ${ }^{13}$ Here $n_{+R}(\mathbf{r})=\bar{n} \Theta(R-r)$ is the density of the positive background. The first term of Eq. (18) is purely a bulk term; it affects nothing calculated in this article, but yields the most realistic bulk moduli with the choice $z=1 .{ }^{13}$ The second term includes an interaction between the electrons and the averaged short-range part of the pseudopotential, which vanishes outside and equals $\langle\delta v\rangle_{W S}$ inside the positive background. Note that $\langle\delta v\rangle_{W S}$ is positive for $r_{s}$ $>4.2$ and negative for $r_{s}<4.2$.

This variational procedure yields approximations to the ground-state densities $n_{R}(x)$ of neutral clusters. For large $N$, the resulting values of the density parameters $d, a$, and $\gamma$ in Eq. (15) can be expanded in the form ${ }^{7}$

$$
d=d_{\infty}+d_{1} N^{-1 / 3}+O\left(N^{-2 / 3}\right)
$$

(and similarly for $a$ and $\gamma$ ), where $d_{\infty}, a_{\infty}$, and $\gamma_{\infty}$ are the corresponding parameters for the density profile of the planar surface. Table I gives the coefficients in these expansions, obtained from least-squares fits to the parameters of clusters with $10000<N<100000$. This table provides model densities for a huge range of cluster sizes for different $r_{s}$ values. 
TABLE II. Comparison of stabilized-jellium results from both Koopmans and $\triangle \mathrm{SCF}$ expressions with direct numerical values from fits to calculated ionization energies of finite clusters. $W$ is the bulk work function, and $c$ is the quantum size-effect coefficient of Eqs. (4) and (1). Very similar results for $c$ are found within the ordinary jellium model (Table II of Ref. 8). $\left(r_{s}\right.$ in bohr, $W$ in eV.)

\begin{tabular}{ccccccc}
\hline \hline$r_{s}$ & $W($ Koopm. $)$ & $W(\Delta \mathrm{SCF})$ & $W($ num. $)$ & $c($ Koopm. $)$ & $c(\Delta \mathrm{SCF})$ & $c$ (num.) \\
\hline 2 & 3.83 & 3.72 & 3.87 & -0.048 & -0.051 & -0.053 \\
3 & 3.62 & 3.27 & 3.29 & -0.041 & -0.074 & -0.079 \\
4 & 3.15 & 2.77 & 2.79 & -0.033 & -0.080 & -0.077 \\
5 & 2.75 & 2.38 & 2.43 & -0.028 & -0.081 & -0.069 \\
6 & 2.45 & 2.10 & 2.09 & -0.023 & -0.079 & -0.080 \\
\hline \hline
\end{tabular}

Note the systematic differences between these parameters and the corresponding ones for ordinary jellium (Table $\mathrm{I}$ in Ref. 7), where $a_{\infty} \approx 0.5 \mathrm{bohr}$ for all different $r_{s}$. In the present case, however, $a_{\infty}$ is strongly $r_{s}$-dependent, indicating that the stabilized-jellium profiles for $r_{s}=2$ are almost twice as steep at the jellium edge as the ones for $r_{s}=6$. This can be seen clearly in Fig. 1(a).

From the coefficients in Table I, we can immediately calculate $^{7}$ the corresponding functions $n(x)$ and $f(x)$ in expansion (6), and thus also $\phi(x)$ and $h(x)$ in Eq. (10). The results for $r_{s}=2,4,6$ are plotted in Fig. 1. We use these functions to evaluate both the $\triangle \mathrm{SCF}$ expressions (7), (8) and the Koopmans expressions (13), (14) for $W$ and $c$. The results are presented in Table II. For $r_{s} \geqslant 3$, the two different types of expressions yield significantly different results, particularly for the coefficient $c$. The reason for this discrepancy is that we are using approximations to the exact functions $n(x)$ and $f(x)$. As we have explained in Ref. 8, the Koopmans expressions are equivalent to the $\triangle \mathrm{SCF}$ expressions if evaluated with the exact functions, but are much more sensitive to small inaccuracies in these functions. [Note that the Koopmans values for $c$, listed in Table II, are also the step heights $\Delta h$ of the functions $h(x)$ in Fig. 1(b), according to Eq. (14).]

To confirm this observation, Table II also contains the direct numerical values for $W$ and $c$, obtained from fitting Eq. (1) to the numerical ionization energies $I(R)=E(N, \nu$ $=-1)-E(N, 0)$, which are calculated as energy differences between positive singly charged and neutral clusters. We call these values "direct" because they involve the energies $E(N,-1)$ of the charged clusters. In contrast, the functions $n(x)$ and $f(x)$, used in evaluating the $\triangle \mathrm{SCF}$ and Koopmans expressions, are extracted from the densities of neutral clusters.

The $\triangle \mathrm{SCF}$ values are strikingly close to the direct numerical values, apart from numerical uncertainties which produce a small oscillation in the latter. The Koopmans values, however, are far off these direct numerical results. For $c$, which is numerically almost equal to the constant -0.08 , the Koopmans values fall close to zero at the lower densities $\left(r_{s}=6\right)$. This is particularly clear from Fig. 2, where the results are presented graphically versus $r_{s}$. Our Koopmans values of $c$ for stabilized jellium differ by less than 0.01 from those of Kiejna and Pogosov. ${ }^{17}$
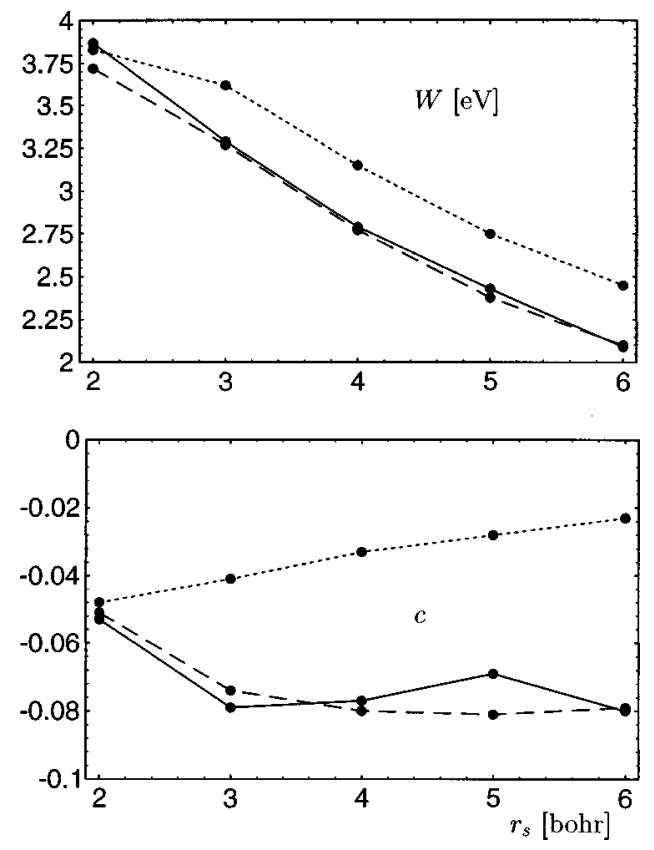

FIG. 2. Work functions $W$ in eV (dots in the upper part of the figure) and size-effect coefficients $c$ (dots in the lower part) for stabilized jellium at $r_{s}=2,3,4,5,6$ bohrs. The dots connected by solid lines represent direct numerical values, obtained from fits to calculated ionization energies. These values are very close to results from the $\Delta \mathrm{SCF}$ expressions (dashed lines). The Koopmans expressions, however, yield very inaccurate results (dotted lines), particularly for $c$.

\section{HOW MANY EXCESS ELECTRONS CAN BE BOUND BY A SIMPLE-METAL CLUSTER?}

In Ref. 26, we presented a continuum or liquid drop model $(\mathrm{LDM})^{7}$ for the energy of a charged spherical cluster with $\nu$ excess electrons (and net charge $-\nu$ ):

$$
E(N, \nu)=E(N, 0)-\nu\left(W+\frac{c}{R+\delta}\right)+\frac{\nu^{2}}{2(R+\delta)},
$$

where $R=r_{s} N^{1 / 3}$ and $N$ is the number of electrons in the neutral $(\nu=0)$ cluster. In Eq. (20), $\delta$ is the distance from the jellium edge to the centroid of infinitesimal excess charge for the planar $(R \rightarrow \infty)$ surface. $\delta$ belongs ${ }^{1}$ in the final or electrostatic term of Eq. (20), where it becomes important for clusters with small radii $R$; our inclusion of it in the second or chemical potential term is purely a matter of convenience, with very little numerical effect. Expanding Eq. (20) in decreasing powers of $N^{1 / 3}$ results in the LDM formula (71) of Ref. 7, if the coefficients $W$ and $c$ are represented by their corresponding $\triangle \mathrm{SCF}$ expressions (7), (8). The distance $\delta$, which is important for small clusters, asymptotically contributes only to terms of order $O\left(N^{-2 / 3}\right)$ and is therefore not relevant in Ref. 7. Eq. (20) neglects shell-structure oscillations, to which we turn in Sec. V.

For integer values of the excess electron number $\nu$ within an open shell, the exact energies $E(N, \nu)$ vs $\nu$ fall more or less upon a smooth curve which is nicely imitated by Eq. (20), as we have indicated in Fig. 3. Thus Eq. (20) not only implies Eqs. (1), (4), and (5), but can be used to estimate higher-order ionization energies and electron affinities. This equation also tells us what to expect for noninteger $\nu$ 


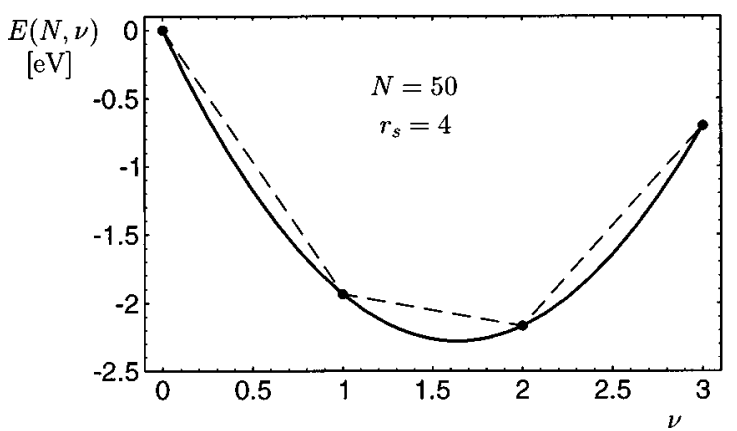

FIG. 3. A plot of the energy $E(N, \nu)$ of Eq. (20) vs excess electron number $\nu$ for a stabilized-jellium cluster with $r_{s}=4$ and $N=50$ (solid curve). We have set $E(N, 0)=0$, and have used for $W, c$, and $\delta$ the same values used to construct Table III. The dashed line models the energy resulting from exact density functional theory, if the dots on the solid curve are presumed to represent the unknown exact energies for integer $\nu$.

within an open shell from a continuous density functional approximation like the local density approximation (LDA).

To bind a given number $\nu$ of excess electrons, the cluster size $N$ must be sufficiently large. Moreover, the condition for the existence of a self-consistent solution to the Kohn-Sham equations, $\partial E(N, \nu) / \partial \nu<0$, in a continuum approximation like LDA where the energy is a smooth function of $\nu$, is more stringent than the condition that the total energy be lowered by the addition of one more electron, $E(N, \nu)$ $-E(N, \nu-1)<0$. This is illustrated in Fig. 3, where the smooth energy curve of Eq. (20) increases with $\nu$ for $\nu$ $>1.6$, although it has its lowest value for integer $\nu$ at $\nu=2$. This curve predicts that a $\mathrm{Na}$ cluster $\left(r_{s} \approx 4\right)$ with $N=50$ atoms can bind $\nu=2$ excess electrons. However, there is no self-consistent LDA solution which localizes 2 excess electrons on the cluster. There is a self-consistent LDA solution which localizes 1.6 excess electrons on the cluster, with the remaining 0.4 electrons distributed over all space.

From Eq. (20), there is a self-consistent solution with $\nu$ excess electrons on the cluster if $N>N_{s c}$, where

$$
N_{s c}=\left(\frac{\nu-c}{W r_{s}}-\frac{\delta}{r_{s}}\right)^{3} \text {, }
$$

but the energy is lower for $\nu$ electrons than for $\nu-1$ when $N>N_{E}$, where

$$
N_{E}=\left(\frac{\nu-c-\frac{1}{2}}{W r_{s}}-\frac{\delta}{r_{s}}\right)^{3},
$$

and clearly $N_{E}<N_{s c}$. Variants of Eq. (22) have been presented in Ref. $27(c=-1 / 8$ and $\delta=0)$ and in Ref. 1 $(c=0)$. Even though there is no self-consistent LDA solution for $N<N_{s c}$, we still predict the existence of stable negative ions with $\nu$ excess electrons for $N>N_{E}$. For $N_{E}<N$ $<N_{s c}$, the LDA solution for integer- $\nu$ excess electrons is a zero-energy resonance, ${ }^{28}$ in which the last excess electron goes into an orbital of zero energy which is partly localized on the cluster and partly delocalized over all of space.

Table III compares $N_{s c}$ and $N_{E}$, Eqs. (21) and (22), for simple-metal clusters, using our most realistic inputs: selfconsistent Kohn-Sham work functions for stabilized jellium (Table IV), $\triangle \mathrm{SCF}$ values for $c$ (Table II), and centroids of
TABLE III. A simple-metal cluster of density parameter $r_{s}$ and neutral radius $R=r_{s} N^{1 / 3}$ can bind $\nu$ excess electrons energetically if $N>N_{E}$, but will find a self-consistent LDA solution for the ion of charge $-\nu$ only for $N>N_{s c}$. Shown are the predictions of the liquid drop model of Eqs. (20)(22) for $N_{s c}$ and $N_{E}$.

\begin{tabular}{ccrr}
\hline \hline$r_{s}$ & $\nu$ & $N_{s c}$ & \multicolumn{1}{c}{$N_{E}$} \\
\hline 2 & 1 & 22.6 & 1.9 \\
2 & 215.0 & 85.7 \\
& 3 & 767.3 & 434.3 \\
& & 10.6 & 1.1 \\
4 & 1 & 92.9 & 38.1 \\
& 2 & 322.6 & 184.6 \\
& 3 & 8.5 & 0.9 \\
& 1 & 73.1 & 30.1 \\
6 & 2 & 252.5 & 144.6 \\
\hline \hline
\end{tabular}

excess charge $\delta$ interpolated from the stabilized-jellium values of Ref. 15: $\delta=1.00,1.11,1.27,1.43$, and $1.60 \mathrm{bohr}$ for $r_{s}=2,3,4,5$, and 6 , respectively.

In the exact density functional theory, ${ }^{12}$ the two different conditions listed in the paragraph before Eq. (21) are equivalent, since the exact energy $E(N, \nu)$ runs linearly between each pair of successive integers $\nu$ and $\nu+1,{ }^{29}$ as shown by the dashed curve in Fig. 3. (Noninteger electron numbers can arise in an average or ensemble sense $\mathrm{e}^{29-31}$ for an open system which exchanges electrons with its environment.) These conditions are not equivalent within the local density approximation, because this approximation makes a selfinteraction error which is larger ${ }^{30,31}$ for noninteger than for integer electron numbers. When a self-interaction correction $^{32}$ is applied, even individual atoms find selfconsistent negative-ion solutions. ${ }^{33}$

For simplicity, we have dicussed only the local density approximation, but the situation is very similar within the local spin density approximation, where the self-interaction error is only slightly smaller, and where the ionization energies of Na clusters (evaluated as total-energy differences) are essentially the same. (Compare Fig. 2 of Ref. 34 with Fig. 4 of the present article.)

Our Eqs. (20)-(22) are relevant to a question raised recently in this journal: ${ }^{18,19}$ Within the local spin density or other continuum approximations, do self-consistent atomlocalized solutions to the Kohn-Sham equations exist for negative atomic ions like $\mathrm{H}^{-}$or $\mathrm{F}^{-}$? When the equations are

TABLE IV. Work functions $W$ from Eq. (7), and surface energies $a_{s}$ from Eq. (9), for stabilized jellium. The results ("s.c.") obtained with the selfconsistent KS density-profile $n^{\mathrm{KS}}(x)$ are compared to the results ("var.") from the restricted variational density profile. Slightly different correlation energy functionals have been used for the "var." and "s.c." calculations. $\left(r_{s}\right.$ in bohr, $W$ and $a_{s}$ in $\mathrm{eV}$.)

\begin{tabular}{ccccc}
\hline \hline$r_{s}$ & $W($ var. $)$ & $W($ s.c. $)$ & $a_{s}($ var. $)$ & $a_{s}$ (s.c.) \\
\hline 2 & 3.72 & 4.30 & 0.694 & 0.868 \\
3 & 3.27 & 3.56 & 0.650 & 0.762 \\
4 & 2.77 & 2.92 & 0.526 & 0.598 \\
5 & 2.38 & 2.47 & 0.429 & 0.475 \\
6 & 2.10 & 2.12 & 0.357 & 0.387 \\
\hline \hline
\end{tabular}




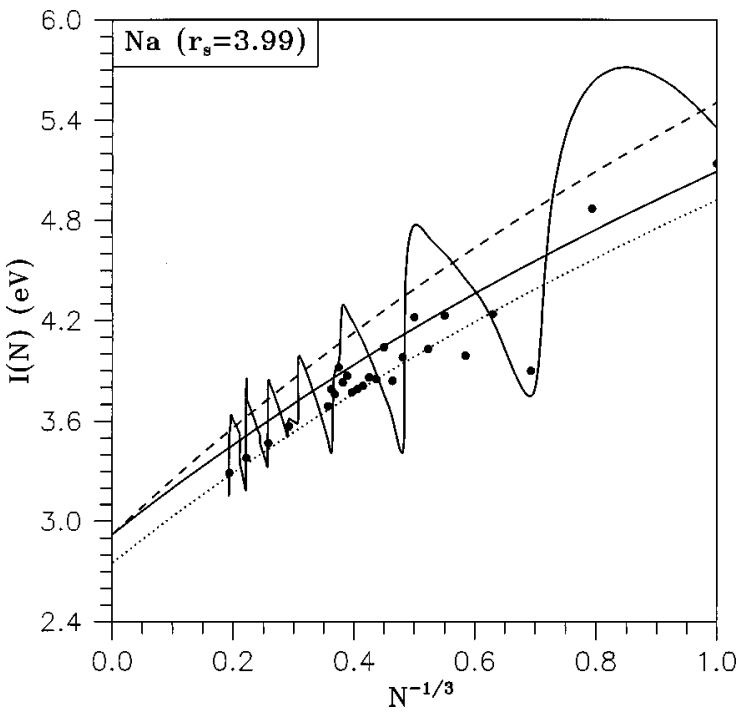

FIG. 4. Ionization energies $I(N)$ (evaluated as total-energy differences) for $r_{s}=3.99$ clusters vs $N^{-1 / 3}$, where $N$ is the number of valence electrons in the neutral cluster. The oscillating solid curve summarizes the Kohn-Sham results for spherical stabilized jellium, and seems to oscillate around the solid line of Eq. (23) (with $\alpha=0.42, \delta=1.27 \mathrm{bohr}$, and $W=2.92 \mathrm{eV}$, the inputs appropriate to stabilized jellium) due to the strong shell-structure effects of spherical models (Refs. 41, 42). The dashed line $(\alpha=0.50)$ is less correct than the solid line $(\alpha=0.42)$. The dots are experimental ionization energies for $\mathrm{Na}$ (Refs. 38, 39, 40) and seem to oscillate around the dotted line of Eq. (23) [with $\alpha=0.42, \delta=1.27 \mathrm{bohr}$, and $W=2.75 \mathrm{eV}=$ the measured polycrystalline work function of $\mathrm{Na}$ (Ref. 38)]. (Na has $r_{s}=3.99$ at room temperature, and 3.93 at absolute zero.)

solved numerically on a radial grid, the answer is no. ${ }^{19,28}$ But, within a finite Gaussian basis (even one that includes diffuse Gaussians), the answer seems to be yes. ${ }^{18}$ We suggest that, to find the proper zero-energy resonance, the basis would have to be flexible enough to admit a Kohn-Sham orbital with two lobes-a large-amplitude lobe on the atom and a diffuse small-amplitude lobe far away-tenuously connected through the potential barrier created by the fractional excess charge on the atom. However, attempts in our group ${ }^{35}$ to find such a zero-energy resonance with the CADPAC ${ }^{36}$ program have not been successful; the extra electron unexpectedly goes into an atom-localized orbital of positive energy.

\section{SELF-CONSISTENT KOHN-SHAM CALCULATIONS, AND A COMPARISON WITH EXPERIMENT}

For small clusters, we have also solved the Kohn-Sham (KS) equations ${ }^{12}$ for the energy functional (16), within the spin-unpolarized local density approximation. Instead of the Perdew-Wang correlation-energy functional, however, we have here used the very similar correlation-energy functional by Vosko, Wilk, and Nusair. ${ }^{37}$ The KS equations do not require an approximation to the unknown functional $T_{s}[n]$ in (16), since the noninteracting kinetic energy is represented by its exact quantum-mechanical operator. Therefore the KS results reflect the microscopic shell effects present in real clusters (cf. Fig. 4). In particular, the KS equations yield self-consistent density profiles $n_{R}^{\mathrm{KS}}(x)$ which are not restricted to a parametrized form such as (15).

Due to the strong shell-effect variations, it is difficult to extract an approximation $f^{\mathrm{KS}}(x)$ to the function $f(x)$ in the expansion (6) from the Kohn-Sham density profiles of small spherical clusters. If it could be so extracted, this function $f^{\mathrm{KS}}(x)$, together with the KS density profile $n^{\mathrm{KS}}(x)$ of the planar metal surface, should yield complete agreement between the Koopmans and $\triangle \mathrm{SCF}$ results for $W$ and $c$.

For sufficiently large radii $R$, the functions $f_{R}^{\mathrm{KS}}(x)$ $\equiv R\left[n_{R}^{\mathrm{KS}}(x)-n^{\mathrm{KS}}(x)\right]$ are expected to approach the exact function $f(x)$ in (6). For clusters with $N<400$, however, these approximations to $f(x)$ still yield very inaccurate $c$ values from Eq. (8) which oscillate strongly about $c \approx$ -0.08 (see Fig. 1 in Ref. 8). Similarly oscillating values result from the expression $c(N) \equiv R[I(R)-W]-\frac{1}{2}$, with the KS ionization energies and work functions inserted for $I(R)$ and $W$.

Unlike the function $f^{\mathrm{KS}}(x)$, the $\mathrm{KS}$ density profile $n^{\mathrm{KS}}(x)$ of the planar metal surface can be calculated directly. ${ }^{11,14}$ Thus for $W$ and $a_{s}$ (but not for $c$ ), we can test the effect of replacing the more realistic $n^{\mathrm{KS}}(x)$ by the restricted-variational density profile for the extended Thomas-Fermi approximation. Table IV shows stabilizedjellium values of the $\triangle \mathrm{SCF}$ expression (7) for the work function $W$, evaluated with the KS density profile $n^{\mathrm{KS}}(x)$ and with the variational profile from the preceding section, respectively. The table also shows stabilized-jellium values $a_{s}^{S J}[n]$ of the surface energy, Eq. (9), obtained from the same density profiles. Although the variational density profile does not show the Friedel oscillations of the more realistic KS profile $n^{\mathrm{KS}}(x)$, the $\Delta \mathrm{SCF}$ expression (7) yields almost the same results for both kinds of profiles.

In order to check our size-effect coefficients of Table II against the predictions of KS theory for small clusters, we have plotted the KS ionization energies of spherical stabilized-jellium clusters with $r_{s}=3.99$ in Fig. 4. For small clusters $(N \approx 1)$, the radius $R$ becomes comparable to the radial centroid of excess charge, e.g., $\delta=1.27 \mathrm{bohr}$ for $r_{s}$ $=4 .{ }^{15}$ Therefore, instead of Eq. (1) we use here the expression

$$
I(R)=W+\frac{\alpha}{R+\delta}, \quad \alpha=\frac{1}{2}+c,
$$

resulting from Eq. (20) by the definition $I(R)=E(N, \nu=$ $-1)-E(N, \nu=0)$. The constant $\delta$ in the denominator of (23) does not affect the value of the asymptotic $R^{-1}$ coefficient $\alpha$ in the expansion (1), but contributes to the higher order $\left(R^{-2}\right)$ terms which become important for small clusters. Figure 4 compares the Kohn-Sham ionization energies (the oscillating solid curve) against the prediction of Eq. (23) (solid line). This figure also compares measured ionization energies of $\mathrm{Na}\left(r_{s}=3.99\right)$ clusters $^{38-40}$ (dots) against Eq. (23) (dotted line).

Two conclusions emerge from Fig. 4: (1) The KohnSham ionization energies of spherical stabilized-jellium clusters execute a strong shell-structure oscillation around the prediction of Eq. (23) (with $\alpha=0.42, \delta=1.27 \mathrm{bohr}$, and $W$ $=2.92 \mathrm{eV}=$ Kohn-Sham work function of stabilized jellium). (2) The measured ionization energies of $\mathrm{Na}$ execute a weaker oscillation around the prediction of Eq. (23) (with $\alpha=0.42$, $\delta=1.27 \mathrm{bohr}$, and $W=2.75 \mathrm{eV}=$ measured work function of $\mathrm{Na}^{38}$. 
Although shell-structure effects are visible in the experimental ionization energies, they are considerably weaker than in the Kohn-Sham results for the stabilized-jellium model because of the artificially high (spherical) symmetry of the model. Ekardt and Penzar ${ }^{41}$ have demonstrated how shell-structure oscillations are reduced in a "distorted droplet model" (see also Ref. 42). We suspect that our Eq. (23) with $\alpha=\frac{1}{2}+c(\Delta \mathrm{SCF}$ ) (and $\delta \neq 0$ ) will produce agreement with experiment whenever the incorrectly derived $\alpha=3 / 8$ (with $\delta=0)$ does, not only for $\mathrm{Na}_{y}(y<138$; Refs. 38, 39, 43), but also for $\mathrm{K}_{y}\left(y<35\right.$; Ref. 43), $\mathrm{Pb}_{y}(y<8$; Ref. 43), $\mathrm{Al}_{y}(y<70$; Ref. 44), etc., where $y=N / z$ and $z$ is the conventional valence.

\section{SUMMARY AND CONCLUSIONS}

We have presented the density profile $n(x)$ of the planar metal surface and its finite-size correction $f(x) / R$ for the profile $n_{R}(x)$ of a finite neutral cluster with radius $R$, extracted from restricted density-variational calculations in the stabilized jellium model. These fundamental functions $n(x)$ and $f(x)$ are the only input to two different but formally exact sets of theoretical expressions for the bulk work function $W$ and for the quantum size-correction coefficient $c$ in the ionization energies $I(R)$ and electron affinities $A(R)$ of finite clusters.

Since we use approximate profiles $n(x)$ and $f(x)$ from restricted density-variational calculations, we can clearly demonstrate that, in the stabilized as in the ordinary jellium model, the $\triangle \mathrm{SCF}$ expressions are highly accurate, i.e., insensitive to errors in the profiles. Unlike the Koopmans expressions, they yield results very close to the "direct" values of $W$ and $c$. These "direct" values are obtained from a numerical fit to the restricted-variational ionization energies of finite clusters, calculated as energy differences between charged and neutral clusters.

The Koopmans and $\triangle \mathrm{SCF}$ expressions would yield identical and appropriate results if their input functions $n(x)$ and $f(x)$ were found from selfconsistent solution of an Euler equation for finite clusters. This might be the Euler equation of the extended Thomas-Fermi problem, or of the KohnSham problem. The most realistic choice is the latter, but its shell-structure oscillations make it very difficult to extract a useful $f(x)$ from Kohn-Sham densities of finite clusters, and we have not been able to do so.

Therefore our method, employing the $\triangle \mathrm{SCF}$ expressions, provides simple access to accurate theoretical ionization energies and electron affinities of large clusters. Remarkably, it does not require any information about the properties of charged clusters, since the functions $n(x)$ and $f(x)$ are obtained from neutral-cluster density profiles.

The stabilized jellium model is expected to be realistic for most simple metals, for which we predict the quantum correction $c \approx-0.08$ in Eqs. (4) and (1) (or -0.05 for the higher-density metals.) Different values of $c$ may well arise for noble or transition metals.

Even for small clusters, our approach provides useful results, if we introduce the radial centroid $R+\delta$ of infinitesimal excess charge, which in a small cluster is significantly different from the radius $R$. From our $W$ and $c$ values, we can then calculate how many excess electrons can be bound by a cluster of given size. In particular, we can explain why some stable negative clusters cannot find selfconsistent solutions within a continuum approximation such as LDA.

\section{ACKNOWLEDGMENTS}

This work was supported in part by the Deutsche Forschungsgemeinschaft, by the U.S. National Science Foundation under Grant No. DMR 95-21353, and by the Junta Nacional de Investigacao Cientifica e Tecnologica (project $\mathrm{PBIC/C/FIS/2216/95)} \mathrm{and} \mathrm{Praxis} \mathrm{XXI} \mathrm{(project} \mathrm{2/2.1/FIS/26/}$ 94).

${ }^{1}$ J. P. Perdew, Phys. Rev. B 37, 6175 (1988).

${ }^{2}$ J. M. Smith, Am. Inst. Aeronaut. Astronaut. J. 3, 648 (1965); D. M. Wood, Phys. Rev. Lett. 46, 749 (1981).

${ }^{3}$ M. Seidl and J. P. Perdew, Phys. Rev. B 50, 5744 (1994).

${ }^{4}$ J. P. Perdew, in Condensed Matter Theories, Vol. 4, edited by J. Keller (Plenum, New York, 1989).

${ }^{5}$ E. Engel and J. P. Perdew, Phys. Rev. B 43, 1331 (1991).

${ }^{6}$ M. Seidl, K.-H. Meiwes-Broer, and M. Brack, J. Chem. Phys. 95, 1295 (1991).

${ }^{7}$ M. Seidl and M. Brack, Ann. Phys. (N.Y.) 245, 275 (1996).

${ }^{8}$ M. Seidl, J. P. Perdew, M. Brajczewska, and C. Fiolhais, Phys. Rev. B 55, 13288 (1997); 57, 4181(E) (1998).

${ }^{9}$ O. D. Häberlen, S.-C. Chung, M. Stener, and N. Rösch, J. Chem. Phys. 106, 5189 (1997).

${ }^{10}$ C. Jackschath, I. Rabin, and W. Schulze, Z. Phys. D 22, 517 (1992).

${ }^{11}$ N. D. Lang and W. Kohn, Phys. Rev. B 3, 1215 (1971).

${ }^{12}$ R. G. Parr and W. Yang, Density-Functional Theory of Atoms and Molecules (Oxford University Press, New York, 1989).

${ }^{13}$ J. P. Perdew, H. Q. Tran, and E. D. Smith, Phys. Rev. B 42, 11627 (1990).

${ }^{14}$ C. Fiolhais and J. P. Perdew, Phys. Rev. B 45, 6207 (1992).

${ }^{15}$ A. Kiejna, Phys. Rev. B 47, 7361 (1993).

${ }^{16}$ I. T. Iakubov and V. V. Pogosov, Physica A 214, 287 (1994).

${ }^{17}$ A. Kiejna and V. V. Pogosov, J. Phys.: Condens. Matter 8, 4245 (1996).

${ }^{18}$ J. M. Galbraith and H. F. Schaefer III, J. Chem. Phys. 105, 862 (1996).

${ }^{19}$ N. Rösch and S. B. Trickey, J. Chem. Phys. 106, 8940 (1997).

${ }^{20}$ M. Brack, Rev. Mod. Phys. 65, 677 (1993).

${ }^{21}$ J. P. Perdew, M. Brajczewska, and C. Fiolhais, Solid State Commun. 88, 795 (1993).

${ }^{22}$ G. D. Mahan and W. L. Schaich, Phys. Rev. B 10, 2647 (1974).

${ }^{23}$ R. Monnier, J. P. Perdew, D. C. Langreth, and J. W. Wilkins, Phys. Rev. B 18, 656 (1978).

${ }^{24}$ M. Brack, Phys. Rev. B 39, 3533 (1989).

${ }^{25}$ J. P. Perdew and Y. Wang, Phys. Rev. B 45, 13244 (1992).

${ }^{26}$ M. Brajczewska, A. Vieira, C. Fiolhais, and J. P. Perdew, Prog. Surf. Sci. 53, 305 (1996).

${ }^{27}$ Y. Ishii, Solid State Commun. 61, 227 (1987).

${ }^{28}$ H. B. Shore, J. H. Rose, and E. Zaremba, Phys. Rev. B 15, 2858 (1977).

${ }^{29}$ J. P. Perdew, R. G. Parr, M. Levy, and J. L. Balduz, Phys. Rev. Lett. 49, 1691 (1982)

${ }^{30}$ J. P. Perdew, in Density Functional Methods in Physics, edited by R. M. Dreizler and J. da Providencia (Plenum, New York, 1985).

${ }^{31}$ J. P. Perdew and M. Levy, Phys. Rev. B 56, 16021 (1997).

${ }^{32}$ J. P. Perdew and A. Zunger, Phys. Rev. B 23, 5048 (1981).

${ }^{33}$ L. A. Cole and J. P. Perdew, Phys. Rev. B 25, 6291 (1982).

${ }^{34}$ M. Brajczewska, C. Fiolhais, and J. P. Perdew, Int. J. Quantum Chem. 27, 249 (1993).

${ }^{35} \mathrm{M}$. Ernzerhof (private communication).

${ }^{36}$ CADPAC6: The Cambridge Analytical Derivatives Package Issue 6.0 Cambridge (1995). A suite for quantum chemistry programs developed by R. D. Amos, with contributions from I. L. Alberts, J. S. Andrews, S. M. Colwell, N. C. Handy, D. Jayatilaka, P. J. Knowles, R. Kobayashi, G. J. Laming, A. M. Lee, P. E. Maslen, C. W. Murray, P. Palmieri, J. E. Rice, J. Sanz, E. D. Simandiras, A. J. Stone, M.-D. Su, and D. J. Tozer. 
${ }^{37}$ S. H. Vosko, L. Wilk, and M. Nusair, Can. J. Phys. 58, 1200 (1980).

${ }^{38}$ E. C. Honea, M. L. Homer, J. L. Persson, and R. L. Whetten, Chem. Phys. Lett. 171, 147 (1990).

${ }^{39}$ M. Kappes, M. Schär, U. Rothlisberger, Ch. Yeretzian, and E. Schumacher, Chem. Phys. Lett. 143, 251 (1988).

${ }^{40}$ C. E. Moore, Ionization Potentials and Ionization Limits Derived from the Analyses of Optical Spectra, Natl. Stand. Ref. Data Ser. (U.S., Natl. Bur.
Stand.) 34, (1970).

${ }^{41}$ W. Ekardt and Z. Penzar, Phys. Rev. B 38, 4273 (1988).

${ }^{42}$ C. Yannouleas and U. Landman, J. Chem. Phys. 107, 1032 (1997).

${ }^{43}$ M. Kappes, M. Schär, P. Radi, and E. Schumacher, J. Chem. Phys. 84, 1863 (1986).

${ }^{44}$ K. E. Schriver, J. L. Persson, E. C. Honea, and R. L. Wilson, Phys. Rev. Lett. 64, 2539 (1990). 